\title{
Mapping Isotopes in Nanoscale and Quantum Materials Using Atom Probe Tomography
}

\author{
Samik Mukherjee ${ }^{1}$, Dieter Isheim ${ }^{2}$, David N. Seidman², Oussama Moutanabbir ${ }^{1}$ \\ 1. Department of Engineering Physics, Polytechnique Montréal, C. P. 6079, Succ. Centre-Ville, \\ Montréal, Quebec, Canada \\ 2. Department of Materials Science and Engineering and Northwestern University Center for Atom- \\ Probe Tomography (NUCAPT), Northwestern University, Evanston, IL, USA
}

Atom probe tomography (APT) with a sub-nanometer scale spatial resolution, supreme mass resolving power, and unparallel analytical sensitivity for 3D compositional imaging at an atomistic level is the only technique capable of probing isotopes in nanoscale materials[1][2]. In this presentation, we discuss the science and practice of atom probe tomographic mapping of isotopes in nanoscale and quantum materials. The introduction of stable isotopes provides an additional degree of freedom to manipulate some basic material properties, highlight subtle but important nanoscale and quantum phenomena, and design an entirely new class of devices with potential application in quantum information processing, band-gap engineering for optoelectronics, nanoelectronics, nano-thermoelectrics, to name a few. For instance, recent measurements showed that isotopically enriched ${ }^{12} \mathrm{C} /{ }^{13} \mathrm{C}$ diamond superlattice can confine charge carriers by a band-gap difference of $17 \mathrm{meV}$ between ${ }^{12} \mathrm{C}$ and ${ }^{13} \mathrm{C}[3]$.

The high evaporation field, limited photoabsorption, strong covalent bonding, and wide bandgap were always known to complicate the field evaporation of diamond, making its control hardly achievable on the atomistic-level. In this work, we demonstrate how the complex interplay of high electric field in a diamond nanotip and its interaction with pulsed laser lead to a controlled field evaporation of atoms thereby enabling 3D atomistic imaging of diamond ${ }^{12} \mathrm{C} /{ }^{13} \mathrm{C}$ homojunctions, as shown in Figure 1a. Our results demonstrates that one key element to achieve controlled evaporation from a diamond nanotip is to operate the pulsed laser at high energy without letting the dc bias increase out of bounds for nanotip to withstand. The role of the dc bias in evaporation of diamond is essentially to generate free charge carriers within the nanotip via impact ionization. The mobile free charges screen the internal electric field, eventually creating a hole rich surface where the pulsed laser energy is effectively absorbed following the laws of free carrier absorption, leading to an increase in the nanotip surface temperature [1]. Also, the complex interplay of nanotip geometry, electric field and the non-uniformity in surface temperature induced by the pulsed laser affects the time-of-flight of an ion (Figure 1b), diffusion of atoms at the nanaotip surface and core (Figure 1c), and distribution of various charged species (Figure 1d) and atomic clusters (Figure 1e) shall be discussed. In addition to paving the way toward a precise manipulation of isotopes in group IV semiconductor-based nanoscale and quantum structures, these results also elucidates some of the basic properties of semiconductor and dielectric nanostructures under high electric field. 


\section{References :}

[1] S. Mukherjee et al., Nano Letters 16 (2016), p.1335.

[2] S. Mukherjee et al., Nano Letters 15 (2015), p. 3885.

[3] H.Watenabe et al., Science 324 (2009), p. 1425

(a)

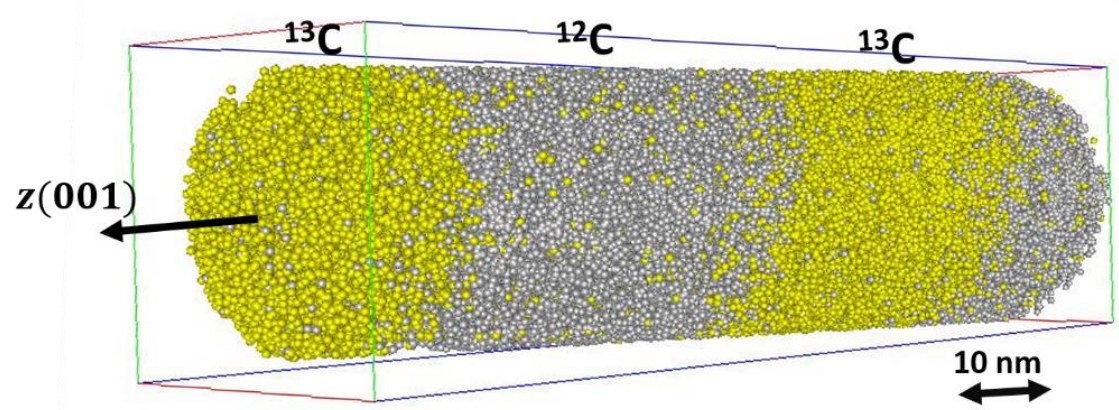

(b)

- ${ }^{13} \mathrm{C}^{2+}$ peak from illuminated surface

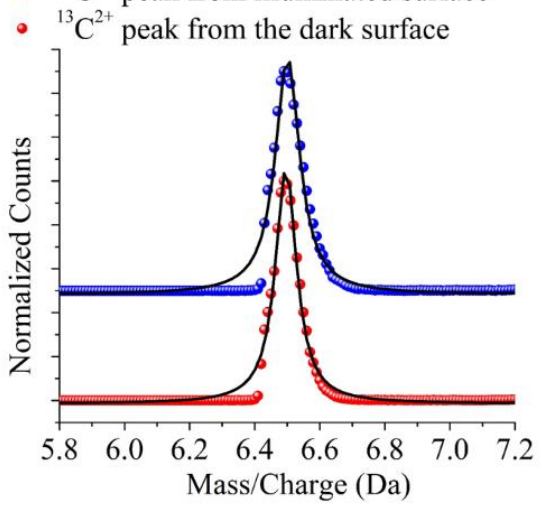

(d)

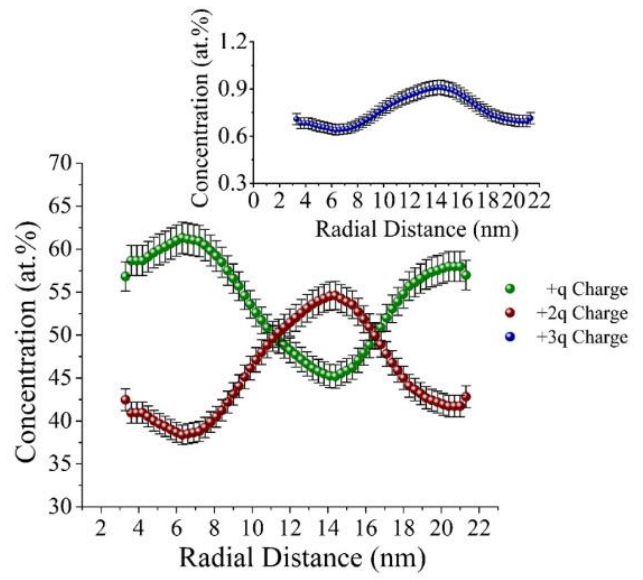

(c)

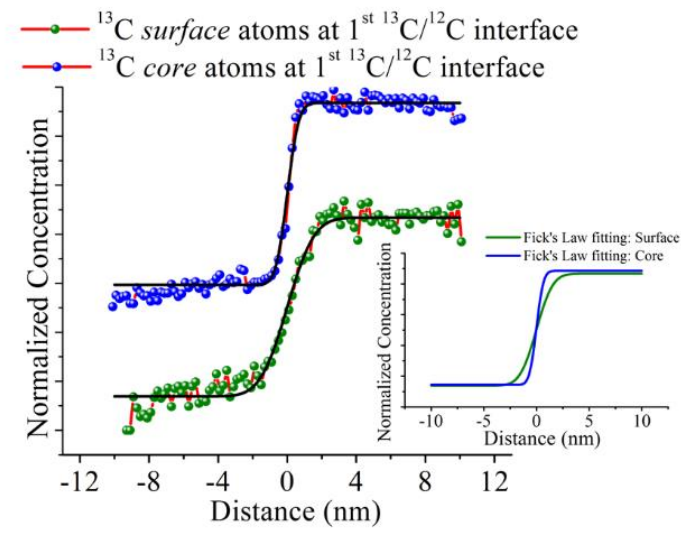

(e)

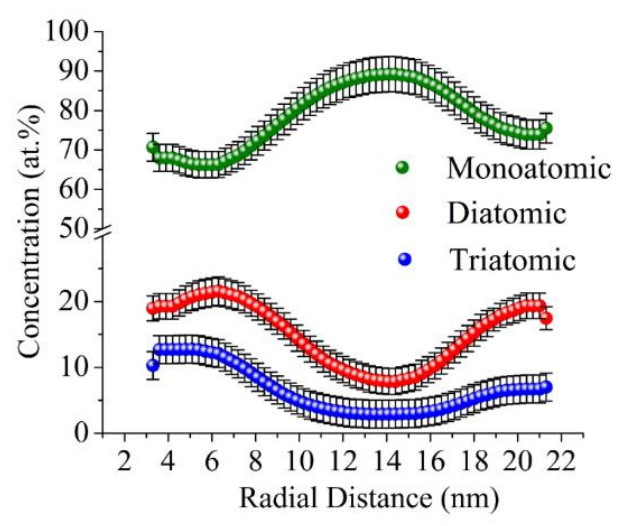

Figure 1. (a) 3D atom by atom reconstruction diamond ${ }^{12} \mathrm{C} /{ }^{13} \mathrm{C}$ isotopic homojunctions (b) Normalized mass spectra of ${ }^{13} \mathrm{C}^{2+}$ atoms located at the surface on the dark side and the illuminated side of the nanotip. The black continuous line is the Lorentzian fit of the data (c) Normalized concentration profile for ${ }^{13} \mathrm{C}$ atoms at the surface and core of the nanotip at the $1^{\text {st }}{ }^{13} \mathrm{C} /{ }^{13} \mathrm{C}$ interface. Inset: Displaying only the Fick's law fitting of the raw data (d) Plot of normalized concentration of $+q,+2 q$ and $+3 q$ (inset) cations with radial diatance (e) Plot of normalized concentration of mono, di, and tri atomic species with radial diatance 